\title{
A KOROVKIN TYPE APPROXIMATION THEOREM FOR DOUBLE SEQUENCES OF POSITIVE LINEAR OPERATORS OF TWO VARIABLES IN $A$-STATISTICAL SENSE
}

\author{
Kamil Demirci and Fadime DiriK
}

\begin{abstract}
In this paper, we obtain a Korovkin type approximation theorem for double sequences of positive linear operators of two variables from $H_{w}(K)$ to $C(K)$ via $A$-statistical convergence. Also, we construct an example such that our new approximation result works but its classical case does not work. Furthermore, we study the rates of $A$-statistical convergence by means of the modulus of continuity.
\end{abstract}

\section{Introduction}

For a sequence $\left(L_{n}\right)$ of positive linear operators on $C(X)$, the space of real valued continuous functions on a compact subset $X$ of real numbers, Korovkin [12] established first the necessary and sufficient conditions for the uniform convergence of $L_{n}(f)$ to a function $f$ by using the test function $e_{i}$ defined by $e_{i}(x)=x^{i},(i=0,1,2)$ (see, for instance, [3]). Later many researchers investigated these conditions for various operators defined on different spaces. Using the concept of statistical convergence in approximation theory provides us with many advantages. In particular, the matrix summability methods of Cesáro type are strong enough to correct the lack of convergence of various sequences of linear operators such as the interpolation operator of HermiteFejér [4], because these types of operators do not converge at points of simple discontinuity. Furthermore, in recent years, with the help of the concept of uniform statistical convergence, which is a regular (non-matrix) summability transformation, various statistical approximation results have been proved [1, $2,6,5,8,11]$. Then, it was demonstrated that those results are more powerful than the classical Korovkin theorem. Also, Erkus and Duman [7] have studied a Korovkin type approximation theorem via $A$-statistical convergence in the space $H_{\omega}\left(I^{2}\right)$ where $I^{2}=[0, \infty) \times[0, \infty)$. Our primary interest in the present

Received March 5, 2009; Revised May 21, 2009.

2000 Mathematics Subject Classification. 41A25, 41A36, 47B38.

Key words and phrases. A-statistical convergence for double sequences, positive linear operator, Korovkin type approximation theorem, Meyer-König and Zeller operator, modulus of continuity. 
paper is to obtain a general Korovkin type approximation theorem for double sequences of positive linear operators of two variables from $H_{\omega}(K)$ to $C(K)$ where $K=[0, A] \times[0, B], A, B \in(0,1)$ via $A$-statistical convergence.

Let us first remind of the concept of $A$-statistical convergence for double sequences.

A double sequence $x=\left(x_{m, n}\right)$ is said to be convergent in Pringsheim's sense if, for every $\varepsilon>0$, there exists $N=N(\varepsilon) \in \mathbb{N}$, the set of all natural numbers, such that $\left|x_{m, n}-L\right|<\varepsilon$ whenever $m, n>N$, where $L$ is called the Pringsheim limit of $x$ and denoted by $P-\lim _{m, n} x_{m, n}=L$ (see [15]). We shall call such an $x$, briefly, "P-convergent". A double sequence is called bounded if there exists a positive number $M$ such that $\left|x_{m, n}\right| \leq M$ for all $(m, n) \in \mathbb{N}^{2}=\mathbb{N} \times \mathbb{N}$. Note that in contrast to the case for single sequences, a convergent double sequence need not to be bounded. Let $A=\left(a_{j, k, m, n}\right)$ be a four-dimensional summability matrix. For a given double sequence $x=\left(x_{m, n}\right)$, the $A$-transform of $x$, denoted by $A x:=\left((A x)_{j, k}\right)$, is given by

$$
(A x)_{j, k}=\sum_{(m, n) \in \mathbb{N}^{2}} a_{j, k, m, n} x_{m, n}
$$

provided the double series converges in Pringsheim's sense for every $(j, k) \in \mathbb{N}^{2}$.

A two dimensional matrix transformation is said to be regular if it maps every convergent sequence in to a convergent sequence with the same limit. The well-known characterization for two dimensional matrix transformations which are regular is known as Silverman-Toeplitz conditions (see, for instance, [10]). In 1926, Robinson [16] presented a four dimensional analog of the regularity by considering an additional assumption of boundedness. This assumption was made because a double $P$-convergent sequence is not necessarily bounded. The definition and the characterization of regularity for four dimensional matrices is known as Robinson-Hamilton conditions, or briefly, $R H$-regularity (see $[9,16]$ ).

Recall that a four dimensional matrix $A=\left(a_{j, k, m, n}\right)$ is said to be $R H$ regular if it maps every bounded $P$-convergent sequence into a $P$-convergent sequence with the same $P$-limit. The Robinson-Hamilton conditions state that a four dimensional matrix $A=\left(a_{j, k, m, n}\right)$ is $R H$-regular if and only if

(i) $P-\lim _{j, k} a_{j, k, m, n}=0$ for each $(m, n) \in \mathbb{N}^{2}$,

(ii) $P-\lim _{j, k} \sum_{(m, n) \in \mathbb{N}^{2}} a_{j, k, m, n}=1$,

(iii) $P-\lim _{j, k} \sum_{m \in \mathbb{N}}\left|a_{j, k, m, n}\right|=0$ for each $n \in \mathbb{N}$,

(iv) $P-\lim _{j, k} \sum_{n \in \mathbb{N}}\left|a_{j, k, m, n}\right|=0$ for each $m \in \mathbb{N}$,

(v) $\sum_{(m, n) \in \mathbb{N}^{2}}\left|a_{j, k, m, n}\right|$ is $P$-convergent, 
(vi) there exist finite positive integers $A$ and $B$ such that $\sum_{m, n>B}\left|a_{j, k, m, n}\right|<$ $A$ holds for every $(j, k) \in \mathbb{N}^{2}$.

Now let $A=\left(a_{j, k, m, n}\right)$ be a non-negative $R H$-regular summability matrix, and let $K \subset \mathbb{N}^{2}$. Then the $A$-density of $K$ is given by

$$
\delta_{A}^{(2)}\{K\}:=P-\lim _{j, k} \sum_{(m, n) \in K(\varepsilon)} a_{j, k, m, n}
$$

where

$$
K(\varepsilon):=\left\{(m, n) \in \mathbb{N}^{2}:\left|x_{m, n}-L\right| \geq \varepsilon\right\}
$$

provided that the limit on the right-hand side exists in Pringsheim's sense. A real double sequence $x=\left(x_{m, n}\right)$ is said to be $A$-statistically convergent to a number $L$ if, for every $\varepsilon>0$,

$$
\delta_{A}^{(2)}\left\{(m, n) \in \mathbb{N}^{2}:\left|x_{m, n}-L\right| \geq \varepsilon\right\}=0 .
$$

In this case, we write $s t_{A}^{(2)}-\lim _{m, n} x=L$. Clearly, a $P$-convergent double sequence is $A$-statistically convergent to the same value but its converse is not always true. Also, note that an $A$-statistically convergent double sequence need not to be bounded. For example, consider the double sequence $x=\left(x_{m, n}\right)$ given by

$$
x_{m, n}= \begin{cases}m n, & \text { if } m \text { and } n \text { are squares, } \\ 0, & \text { otherwise. }\end{cases}
$$

We should note that if we take $A=C(1,1)$, which is the double Cesáro matrix, then $C(1,1)$-statistical convergence coincides with the notion of statistical convergence for double sequence, which was introduced in $[13,14]$ : If $E \subset \mathbb{N}^{2}$ is a two-dimensional subset of positive integers, then $E_{j, k}$ denotes the set $\{(m, n) \in E: m \leq j, n \leq k\}$ and $\left|E_{j, k}\right|$ denotes the cardinality of $E_{j, k}$. The double natural density of $E[13,14]$ is given by $\delta^{(2)}(E):=$ $P-\lim _{j, k} \frac{1}{j k}\left|E_{j, k}\right|$, if it exists. The number sequence $x=\left(x_{m, n}\right)$ is statistically convergent to $L$ provided that for every $\varepsilon>0$, the set $E:=E(\varepsilon):=$ $\left\{m \leq j, n \leq k:\left|x_{m, n}-L\right| \geq \varepsilon\right\}$ has natural density zero; in that case we write $s t^{(2)}-\lim _{m, n} x_{m, n}=L$.

Finally, if we replace the matrix $A$ by the identity matrix for four dimensional matrices, then $A$-statistical convergence reduces to the Pringsheim convergence.

\section{A Korovkin-type approximation theorem}

Throughout this section let $I=[0, A], J=[0, B], A, B \in(0,1)$ and $K=$ $I \times J$. We denote by $C(K)$ the space of all continuous real valued functions on $K$. This space is equipped with the supremum norm

$$
\|f\|=\sup _{(x, y) \in K}|f(x, y)|,(f \in C(K)) .
$$


Consider the space $H_{\omega}(K)$ of all real valued functions $f$ on $K$ and satisfying

$$
|f(u, v)-f(x, y)| \leq \omega\left(f ; \sqrt{\left(\frac{u}{1-u}-\frac{x}{1-x}\right)^{2}+\left(\frac{v}{1-v}-\frac{y}{1-y}\right)^{2}}\right)
$$

where $\omega$ is the modulus of continuity given by, for $\delta>0$,

$$
\omega(f ; \delta):=\sup \left\{|f(u, v)-f(x, y)|:(u, v),(x, y) \in K, \sqrt{(u-x)^{2}+(v-y)^{2}} \leq \delta\right\} .
$$

Then observe that any function in $H_{\omega}(K)$ is continuous and bounded on $K$. We also use the following test functions

$$
\begin{aligned}
& f_{0}(u, v)=1, f_{1}(u, v)=\frac{u}{1-u}, f_{2}(u, v)=\frac{v}{1-v} \text { and } \\
& f_{3}(u, v)=\left(\frac{u}{1-u}\right)^{2}+\left(\frac{v}{1-v}\right)^{2} .
\end{aligned}
$$

Now we have the following result.

Theorem 1. Let $\left\{L_{m, n}\right\}$ be a sequence of positive linear operators from $H_{\omega}(K)$ into $C(K)$ and let $A=\left(a_{j, k, m, n}\right)$ be a nonnegative $R H$-regular summability matrix. Assume that the following conditions hold:

$$
s t_{A}^{(2)}-\lim _{m, n}\left\|L_{m, n}\left(f_{i}\right)-f_{i}\right\|=0, i=0,1,2,3 .
$$

Then, for any $f \in H_{\omega}(K)$,

$$
s t_{A}^{(2)}-\lim _{m, n}\left\|L_{m, n}(f)-f\right\|=0 .
$$

Proof. Assume that (2.1) holds. Let $f \in H_{\omega}(K)$ and $(x, y) \in K$ be fixed. After some simple calculations, using the continuity of $f$ and linearity and positivity of the operators $L_{m, n}$, we obtain

$$
\begin{aligned}
& \left|L_{m, n}(f ; x, y)-f(x, y)\right| \\
\leq & C\left\{\left|L_{m, n}\left(f_{0} ; x, y\right)-f_{0}(x, y)\right|+\left|L_{m, n}\left(f_{1} ; x, y\right)-f_{1}(x, y)\right|\right. \\
& \left.\left|L_{m, n}\left(f_{2} ; x, y\right)-f_{2}(x, y)\right|+\left|L_{m, n}\left(f_{3} ; x, y\right)-f_{3}(x, y)\right|\right\}+\varepsilon,
\end{aligned}
$$

where $C:=\max \left\{\varepsilon+N+\frac{2 N}{\delta^{2}}\left(\left(\frac{A}{1-A}\right)^{2}+\left(\frac{B}{1-B}\right)^{2}\right), \frac{4 N}{\delta^{2}} \frac{A}{1-A}, \frac{4 N}{\delta^{2}} \frac{B}{1-B}, \frac{2 N}{\delta^{2}}\right\}$ and $N:=\|f\|$. Then, taking supremum over $(x, y) \in K$ we get

$$
\left\|L_{m, n}(f)-f\right\| \leq \varepsilon+C \sum_{i=0}^{3}\left\|L_{m, n}\left(f_{i}\right)-f_{i}\right\| .
$$

For a given $r>0$, choose $\varepsilon>0$ such that $\varepsilon<r$. Then, for each $i=0,1,2,3$, setting

$$
U:=\left\{(m, n):\left\|L_{m, n}(f)-f\right\| \geq r\right\}
$$


and

$$
U_{i}:=\left\{(m, n):\left\|L_{m, n}\left(f_{i}\right)-f_{i}\right\| \geq \frac{r-\varepsilon}{4 C}\right\}, i=0,1,2,3,
$$

it follows from (2.3) that

$$
U \subset \bigcup_{i=0}^{3} U_{i}
$$

which gives, for all $(j, k) \in \mathbb{N}^{2}$,

$$
\sum_{(m, n) \in U} a_{j, k, m, n} \leq \sum_{i=0}^{3} \sum_{(m, n) \in U_{i}} a_{j, k, m, n}
$$

Letting $j, k \rightarrow \infty$ (in any manner) and using (2.1), we obtain (2.2). The proof is complete.

Remark 1 . If we replace the matrix $A$ in Theorem 1 by identity double matrix, then we immediately get the following classical result, which was first introduced by Taşdelen and Erençin [17].

Corollary $1([17])$. Let $\left\{L_{m, n}\right\}$ be a sequence of positive linear operators from $H_{\omega}(K)$ into $C(K)$. Assume that the following conditions hold:

$$
P-\lim _{m, n}\left\|L_{m, n}\left(f_{i}\right)-f_{i}\right\|=0, i=0,1,2,3 .
$$

Then, for any $f \in H_{\omega}(K)$,

$$
P-\lim _{m, n}\left\|L_{m, n}(f)-f\right\|=0 .
$$

Remark 2 . We now show that our result Theorem 1 is stronger than its classical version Corollary 1. To see this first consider the following Meyer-König and Zeller operators:

$$
\begin{aligned}
& M_{m, n}(f ; x, y) \\
= & (1-x)^{m+1}(1-y)^{n+1} \sum_{k=0}^{\infty} \sum_{l=0}^{\infty} f\left(\frac{k}{k+m+1}, \frac{l}{l+n+1}\right)\left(\begin{array}{c}
m+k \\
k
\end{array}\right)\left(\begin{array}{c}
n+l \\
l
\end{array}\right) x^{k} y^{l},
\end{aligned}
$$

where $f \in H_{\omega}(K)$, and $K=[0, A] \times[0, B], A, B \in(0,1)$.

Since, for $x \in[0, A], A \in(0,1)$,

$$
\frac{1}{(1-x)^{m+1}}=\sum_{k=0}^{\infty}\left(\begin{array}{c}
m+k \\
k
\end{array}\right) x^{k}
$$

it is clear that, for all $(m, n) \in \mathbb{N}^{2}$,

$$
M_{m, n}\left(f_{0} ; x, y\right)=f_{0}(x, y) .
$$


Also we obtain

$$
\begin{aligned}
M_{m, n}\left(f_{1} ; x, y\right) & =(1-x)^{m+1}(1-y)^{n+1} \sum_{k=0}^{\infty} \sum_{l=0}^{\infty} \frac{k}{m+1}\left(\begin{array}{c}
m+k \\
k
\end{array}\right)\left(\begin{array}{c}
n+l \\
l
\end{array}\right) x^{k} y^{l} \\
& =(1-x)^{m+1}(1-y)^{n+1} x \sum_{k=1}^{\infty} \sum_{l=0}^{\infty} \frac{1}{m+1} \frac{(m+k) !}{m !(k-1) !}\left(\begin{array}{c}
n+l \\
l
\end{array}\right) x^{k-1} y^{l} \\
& =(1-x)^{m+1}(1-y)^{n+1} x \frac{1}{(1-x)^{m+2}} \frac{1}{(1-y)^{n+1}} \\
& =\frac{x}{1-x}
\end{aligned}
$$

and, similarly,

$$
\begin{aligned}
M_{m, n}\left(f_{2} ; x, y\right) & =(1-x)^{m+1}(1-y)^{n+1} \sum_{k=0}^{\infty} \sum_{l=0}^{\infty} \frac{l}{n+1}\left(\begin{array}{c}
m+k \\
k
\end{array}\right)\left(\begin{array}{c}
n+l \\
l
\end{array}\right) x^{k} y^{l} \\
& =\frac{y}{1-y} .
\end{aligned}
$$

Finally we get

$$
\begin{aligned}
& M_{m, n}\left(f_{3} ; x, y\right) \\
& =(1-x)^{m+1}(1-y)^{n+1} \sum_{k=0}^{\infty} \sum_{l=0}^{\infty}\left\{\left(\frac{k}{m+1}\right)^{2}+\left(\frac{l}{n+1}\right)^{2}\right\}\left(\begin{array}{c}
m+k \\
k
\end{array}\right)\left(\begin{array}{c}
n+l \\
l
\end{array}\right) x^{k} y^{l} \\
& =(1-x)^{m+1}(1-y)^{n+1} \sum_{k=0}^{\infty} \sum_{l=0}^{\infty}\left(\frac{k}{m+1}\right)^{2}\left(\begin{array}{c}
m+k \\
k
\end{array}\right)\left(\begin{array}{c}
n+l \\
l
\end{array}\right) x^{k} y^{l} \\
& +(1-x)^{m+1}(1-y)^{n+1} \sum_{k=0}^{\infty} \sum_{l=0}^{\infty}\left(\frac{l}{n+1}\right)^{2}\left(\begin{array}{c}
m+k \\
k
\end{array}\right)\left(\begin{array}{c}
n+l \\
l
\end{array}\right) x^{k} y^{l} \\
& =(1-x)^{m+1}(1-y)^{n+1} \frac{x}{m+1} \sum_{k=1}^{\infty} \sum_{l=0}^{\infty} \frac{k}{m+1} \frac{(m+k) !}{m !(k-1) !}\left(\begin{array}{c}
n+l \\
l
\end{array}\right) x^{k-1} y^{l} \\
& +(1-x)^{m+1}(1-y)^{n+1} \frac{y}{n+1} \sum_{k=0}^{\infty} \sum_{l=1}^{\infty} \frac{l}{n+1}\left(\begin{array}{c}
m+k \\
k
\end{array}\right) \frac{(n+l) !}{n !(l-1) !} x^{k} y^{l-1} \\
& =(1-x)^{m+1}(1-y)^{n+1} \frac{x}{m+1}\left\{x \sum_{k=1}^{\infty} \sum_{l=0}^{\infty} \frac{(m+k+1) !}{(m+1) !(k-1) !}\left(\begin{array}{c}
n+l \\
l
\end{array}\right) x^{k-1} y^{l}\right. \\
& \left.+\sum_{k=0}^{\infty} \sum_{l=0}^{\infty}\left(\begin{array}{c}
m+k+1 \\
k
\end{array}\right)\left(\begin{array}{c}
n+l \\
l
\end{array}\right) x^{k} y^{l}\right\} \\
& +(1-x)^{m+1}(1-y)^{n+1} \frac{y}{n+1}\left\{\sum_{k=0}^{\infty} \sum_{l=1}^{\infty}\left(\begin{array}{c}
m+k \\
k
\end{array}\right) \frac{(n+l+1) !}{(n+1) !(l-1) !} x^{k} y^{l-1}\right. \\
& \left.+\sum_{k=0}^{\infty} \sum_{l=0}^{\infty}\left(\begin{array}{c}
m+k \\
k
\end{array}\right)\left(\begin{array}{c}
n+l+1 \\
l
\end{array}\right) x^{k} y^{l}\right\}
\end{aligned}
$$


$=\frac{m+2}{m+1}\left(\frac{x}{1-x}\right)^{2}+\frac{1}{m+1} \frac{x}{1-x}+\frac{n+2}{n+1}\left(\frac{y}{1-y}\right)^{2}+\frac{1}{n+1} \frac{y}{1-y}$.

Hence, by Corollary 1 , we know that, for any $f \in H_{\omega}(K)$,

$$
P-\lim _{m, n}\left\|M_{m, n}(f)-f\right\|=0 .
$$

Now take $A=C(1,1)$ and define a double sequence $\left\{u_{m, n}\right\}$ by

$$
u_{m, n}= \begin{cases}1, & \text { if } m \text { and } n \text { are squares } \\ 0, & \text { otherwise. }\end{cases}
$$

In this case, observe that

$$
s t_{C(1,1)}^{(2)}-\lim _{m, n} u_{m, n}=0 .
$$

However, the sequence $\left(u_{m, n}\right)$ is not $P$-convergent. Now using $(2.4)$ and (2.6), we define the following positive linear operators on $H_{\omega}(K)$ as follows:

$$
L_{m, n}(f ; x, y)=\left(1+u_{m, n}\right) M_{m, n}(f ; x, y) .
$$

Observe that

$$
\begin{aligned}
\left\|L_{m, n}\left(f_{0}\right)-f_{0}\right\| & =u_{m, n} \\
\left\|L_{m, n}\left(f_{1}\right)-f_{1}\right\| & =u_{m, n} \\
\left\|L_{m, n}\left(f_{2}\right)-f_{2}\right\| & =u_{m, n} \\
\left\|L_{m, n}\left(f_{3}\right)-f_{3}\right\| & =\|\left(1+u_{m, n}\right)\left(\frac{m+2}{m+1}\left(\frac{x}{1-x}\right)^{2}+\frac{1}{m+1} \frac{x}{1-x}\right. \\
& \left.+\frac{n+2}{n+1}\left(\frac{y}{1-y}\right)^{2}+\frac{1}{n+1} \frac{y}{1-y}\right) \\
& \quad-\left(\frac{x}{1-x}\right)^{2}-\left(\frac{y}{1-y}\right)^{2} \| \\
\leq D & \left\{\frac{2}{m+1}+\frac{2}{n+1}+u_{m, n} \frac{m+3}{m+1}+u_{m, n} \frac{n+3}{n+1}\right\},
\end{aligned}
$$

where $D:=\left\{\left(\frac{A}{1-A}\right)^{2},\left(\frac{B}{1-B}\right)^{2}, \frac{A}{1-A}, \frac{B}{1-B}\right\}$. Then, since $s t_{C(1,1)}^{(2)}-\lim _{m, n} u_{m, n}$ $=0$, we obtain $s t_{C(1,1)}^{(2)}-\lim _{m, n}\left\|L_{m, n}\left(f_{i}\right)-f_{i}\right\|=0, i=0,1,2$. Now, for a given $\varepsilon>0$, it follows from above inequality that

$$
\begin{aligned}
& \frac{1}{j k}\left|\left\{m \leq j, n \leq k:\left\|L_{m, n}\left(f_{3}\right)-f_{3}\right\| \geq \varepsilon\right\}\right| \\
\leq & \frac{1}{j k}\left|\left\{m \leq j, n \leq k: \frac{1}{m+1} \geq \frac{\varepsilon}{8 D}\right\}\right| \\
+ & \frac{1}{j k}\left|\left\{m \leq j, n \leq k: \frac{1}{n+1} \geq \frac{\varepsilon}{8 D}\right\}\right|
\end{aligned}
$$




$$
\begin{aligned}
& +\frac{1}{j k}\left|\left\{m \leq j, n \leq k: u_{m, n} \frac{m+3}{m+1} \geq \frac{\varepsilon}{4 D}\right\}\right| \\
& +\frac{1}{j k}\left|\left\{m \leq j, n \leq k: u_{m, n} \frac{n+3}{n+1} \geq \frac{\varepsilon}{4 D}\right\}\right| .
\end{aligned}
$$

Since $s t_{C(1,1)}^{(2)}-\lim _{m, n} u_{m, n}=0$, letting $j, k \rightarrow \infty$ (in any manner) we obtain $s t_{C(1,1)}^{(2)}-\lim _{m, n}\left\|L_{m, n}\left(f_{3}\right)-f_{3}\right\|=0$. Hence, the sequence of positive linear operators $\left\{L_{m, n}\right\}$ defined by (2.8) satisfies all hypotheses of Theorem 1. So, by (2.5) and (2.7), we have

$$
s t_{C(1,1)}^{(2)}-\lim _{m, n}\left\|L_{m, n}(f)-f\right\|=0 .
$$

However, since $\left(u_{m, n}\right)$ is not $P$-convergent, the sequence $\left\{L_{m, n}(f ; x, y\}\right.$ given by (2.8) does not converge uniformly to the function $f \in H_{\omega}(K)$. So, we conclude that Corollary 1 does not work for the operators $L_{m, n}$ in (2.8) while our Theorem 1 still works.

\section{Rate of $\boldsymbol{A}$-statistical convergence}

Various ways of defining rates of convergence in the $A$-statistical sense for two-dimensional summability matrices were introduced in [6]. In a similar way, for four-dimensional summability matrices, we present four different ways to compute the corresponding rates of $A$-statistical convergence in Theorem 1.

Definition 1. Let $A=\left(a_{j, k, m, n}\right)$ be a non-negative $R H$-regular summability matrix and let $\left\{\alpha_{m, n}\right\}$ be a positive non-increasing double sequence. A double sequence $x=\left\{x_{m, n}\right\}$ is $A$-statistically convergent to a number $L$ with the rate of $o\left(\alpha_{m, n}\right)$ if for every $\varepsilon>0$,

$$
P-\lim _{j, k \rightarrow \infty} \frac{1}{\alpha_{j, k}} \sum_{(m, n) \in K(\varepsilon)} a_{j, k, m, n}=0
$$

where

$$
K(\varepsilon):=\left\{(m, n) \in \mathbb{N}^{2}:\left|x_{m, n}-L\right| \geq \varepsilon\right\} .
$$

In this case, we write

$$
x_{m, n}-L=s t_{A}^{(2)}-o\left(\alpha_{m, n}\right) \text { as } m, n \rightarrow \infty .
$$

Definition 2. Let $A=\left(a_{j, k, m, n}\right)$ and $\left\{\alpha_{m, n}\right\}$ be the same as in Definition 1 . Then, a double sequence $x=\left\{x_{m, n}\right\}$ is $A$-statistically bounded with the rate of $O\left(\alpha_{m, n}\right)$ if for every $\varepsilon>0$,

$$
\sup _{j, k} \frac{1}{\alpha_{j, k}} \sum_{(m, n) \in L(\varepsilon)} a_{j, k, m, n}<\infty,
$$

where

$$
L(\varepsilon):=\left\{(m, n) \in \mathbb{N}^{2}:\left|x_{m, n}\right| \geq \varepsilon\right\} .
$$


In this case, we write

$$
x_{m, n}=s t_{A}^{(2)}-O\left(\alpha_{m, n}\right) \text { as } m, n \rightarrow \infty .
$$

Definition 3. Let $A=\left(a_{j, k, m, n}\right)$ and $\left\{\alpha_{m, n}\right\}$ be the same as in Definition 1. Then, a double sequence $x=\left\{x_{m, n}\right\}$ is $A$-statistically convergent to a number $L$ with the rate of $o_{m}\left(\alpha_{m, n}\right)$ if for every $\varepsilon>0$,

$$
P-\lim _{j, k \rightarrow \infty} \sum_{(m, n) \in M(\varepsilon)} a_{j, k, m, n}=0,
$$

where

$$
M(\varepsilon):=\left\{(m, n) \in \mathbb{N}^{2}:\left|x_{m, n}-L\right| \geq \varepsilon \alpha_{m, n}\right\} .
$$

In this case, we write

$$
x_{m, n}-L=s t_{A}^{(2)}-o_{m}\left(\alpha_{m, n}\right) \text { as } m, n \rightarrow \infty .
$$

Definition 4. Let $A=\left(a_{j, k, m, n}\right)$ and $\left\{\alpha_{m, n}\right\}$ be the same as in Definition 1. Then, a double sequence $x=\left\{x_{m, n}\right\}$ is $A$-statistically bounded with the rate of $O_{m}\left(\alpha_{m, n}\right)$ if for every $\varepsilon>0$,

$$
P-\lim _{j, k} \sum_{(m, n) \in N(\varepsilon)} a_{j, k, m, n}=0,
$$

where

$$
N(\varepsilon):=\left\{(m, n) \in \mathbb{N}^{2}:\left|x_{m, n}\right| \geq \varepsilon \alpha_{m, n}\right\} .
$$

In this case, we write

$$
x_{m, n}-L=s t_{A}^{(2)}-O_{m}\left(\alpha_{m, n}\right) \text { as } m, n \rightarrow \infty .
$$

We see from the above statements that, in Definitions 1 and 2 the rate sequence $\left\{a_{m, n}\right\}$ directly effects the entries of the matrix $A=\left(a_{j, k, m, n}\right)$ although, according to Definitions 3 and 4 , the rate is more controlled by the terms of the sequence $x=\left\{x_{m, n}\right\}$.

Using these definitions we obtain the following auxiliary result.

Lemma 1. Let $\left\{x_{m, n}\right\}$ and $\left\{y_{m, n}\right\}$ be double sequences. Assume that $A=$ $\left(a_{j, k, m, n}\right)$ is a non-negative $R H$-regular summability matrix, and let $\left\{\alpha_{m, n}\right\}$ and $\left\{\beta_{m, n}\right\}$ be positive non-increasing sequences. If $x_{m, n}-L_{1}=s t_{A}^{(2)}-o\left(\alpha_{m, n}\right)$ and $y_{m, n}-L_{2}=s t_{A}^{(2)}-o\left(\beta_{m, n}\right)$, then we have

(i) $\left(x_{m, n}-L_{1}\right) \mp\left(y_{m, n}-L_{2}\right)=s t_{A}^{(2)}-o\left(\gamma_{m, n}\right)$ as $m, n \rightarrow \infty$, where $\gamma_{m, n}:=\max \left\{\alpha_{m, n}, \beta_{m, n}\right\}$ for each $(m, n) \in \mathbb{N}^{2}$,

(ii) $\lambda\left(x_{m, n}-L_{1}\right)=s t_{A}^{(2)}-o\left(\alpha_{m, n}\right)$ as $m, n \rightarrow \infty$ for any real number $\lambda$.

Furthermore, similar conclusions hold with the symbol "o" replaced by " $O$ ".

Proof. (i) Assume that $x_{m, n}-L_{1}=s t_{A}^{(2)}-o\left(\alpha_{m, n}\right)$ and $y_{m, n}-L_{2}=s t_{A}^{(2)}-$ $o\left(\beta_{m, n}\right)$. Also, for $\varepsilon>0$, define

$$
K:=\left\{(m, n) \in \mathbb{N}^{2}:\left|\left(x_{m, n}-L_{1}\right) \mp\left(y_{m, n}-L_{2}\right)\right| \geq \varepsilon\right\},
$$




$$
\begin{aligned}
& K_{1}:=\left\{(m, n) \in \mathbb{N}^{2}:\left|x_{m, n}-L_{1}\right| \geq \frac{\varepsilon}{2}\right\}, \\
& K_{2}:=\left\{(m, n) \in \mathbb{N}^{2}:\left|y_{m, n}-L_{2}\right| \geq \frac{\varepsilon}{2}\right\} .
\end{aligned}
$$

Then observe that

$$
K \subset K_{1} \cup K_{2}
$$

which gives, for all $(j, k) \in \mathbb{N}^{2}$,

$$
\sum_{(m, n) \in K} a_{j, k, m, n} \leq \sum_{(m, n) \in K_{1}} a_{j, k, m, n}+\sum_{(m, n) \in K_{2}} a_{j, k, m, n} .
$$

Since $\gamma_{m, n}=\max \left\{\alpha_{m, n}, \beta_{m, n}\right\}$, by (3.1), we get

$$
\frac{1}{\gamma_{j, k}} \sum_{(m, n) \in K} a_{j, k, m, n} \leq \frac{1}{\alpha_{j, k}} \sum_{(m, n) \in K_{1}} a_{j, k, m, n}+\frac{1}{\beta_{j, k}} \sum_{(m, n) \in K_{2}} a_{j, k, m, n} .
$$

Now by taking the limit as $j, k \rightarrow \infty$ (in any manner) in (3.2) and using the hypotheses, we conclude that

$$
P-\lim _{j, k \rightarrow \infty} \frac{1}{\gamma_{j, k}} \sum_{(m, n) \in K} a_{j, k, m, n}=0,
$$

which completes the proof of (i). Since the proof of (ii) is similar, we omit it.

The above proof can easily be modified to prove the following analogue.

Lemma 2. Let $\left\{x_{m, n}\right\}$ and $\left\{y_{m, n}\right\}$ be double sequences. Assume that $A=$ $\left(a_{j, k, m, n}\right)$ is a non-negative $R H$-regular summability matrix, and let $\left\{\alpha_{m, n}\right\}$ and $\left\{\beta_{m, n}\right\}$ be positive non-increasing sequences. If $x_{m, n}-L_{1}=s t_{A}^{(2)}-o_{m}\left(\alpha_{m, n}\right)$ and $y_{m, n}-L_{2}=s t_{A}^{(2)}-o_{m}\left(\beta_{m, n}\right)$, then we have

(i) $\left(x_{m, n}-L_{1}\right) \mp\left(y_{m, n}-L_{2}\right)=s t_{A}^{(2)}-o_{m}\left(\gamma_{m, n}\right)$ as $m, n \rightarrow \infty$, where $\gamma_{m, n}:=\max \left\{\alpha_{m, n}, \beta_{m, n}\right\}$ for each $(m, n) \in \mathbb{N}^{2}$,

(ii) $\lambda\left(x_{m, n}-L_{1}\right)=s t_{A}^{(2)}-o_{m}\left(\alpha_{m, n}\right)$ as $m, n \rightarrow \infty$ for any real number $\lambda$. Furthermore, similar conclusions hold with the symbol " $o_{m}$ " replaced by " $O_{m}$ ".

Now we have the following result.

Theorem 2. Let $\left\{L_{m, n}\right\}$ be a sequence of positive linear operators from $H_{\omega}(K)$ into $C(K)$, and let $A=\left(a_{j, k, m, n}\right)$ be a nonnegative $R H$-regular summability matrix method. Assume that the following conditions hold:

(i) $\left\|L_{m, n}\left(f_{0}\right)-f_{0}\right\|=s t_{A}^{(2)}-o\left(\alpha_{m, n}\right)$ as $m, n \rightarrow \infty$,

(ii) $\omega\left(f ; \delta_{m, n}\right)=s t_{A}^{(2)}-o\left(\beta_{m, n}\right)$ as $m, n \rightarrow \infty$, where $\delta_{m, n}:=\sqrt{\left\|L_{m, n}(\varphi)\right\|}$ with $\varphi(u, v)=\left(\frac{u}{1-u}-\frac{x}{1-x}\right)^{2}+\left(\frac{v}{1-v}-\frac{y}{1-y}\right)^{2}$. Then, for any $f \in H_{\omega}(K)$,

$$
\left\|L_{m, n}(f)-f\right\|=s t_{A}^{(2)}-o\left(\gamma_{m, n}\right) \text { as } m, n \rightarrow \infty,
$$


where $\gamma_{m, n}:=\max \left\{\alpha_{m, n}, \beta_{m, n}\right\}$ for each $(m, n) \in \mathbb{N}^{2}$. Furthermore, similar results holds when the symbol "o" is replaced by "O".

Proof. Let $f \in H_{\omega}(K)$ and $(x, y) \in K$ be fixed. Using linearity and positivity of the $L_{m, n}$, we have, for any $(m, n) \in \mathbb{N}^{2}$,

$$
\begin{aligned}
& \left|L_{m, n}(f ; x, y)-f(x, y)\right| \\
= & \left|L_{m, n}(f(u, v)-f(x, y) ; x, y)-f(x, y)\left(L_{m, n}\left(f_{0} ; x, y\right)-f_{0}(x, y)\right)\right| \\
\leq & L_{m, n}(|f(u, v)-f(x, y)| ; x, y)+N\left|L_{m, n}\left(f_{0} ; x, y\right)-f_{0}(x, y)\right| \\
\leq & \omega(f ; \delta) L_{m, n}\left(1+\frac{\sqrt{\left(\frac{u}{1-u}-\frac{x}{1-x}\right)^{2}+\left(\frac{v}{1-v}-\frac{y}{1-y}\right)^{2}}}{\delta} ; x, y\right) \\
& +N\left|L_{m, n}\left(f_{0} ; x, y\right)-f_{0}(x, y)\right| \\
\leq & \omega(f ; \delta)\left|L_{m, n}\left(f_{0} ; x, y\right)-f_{0}(x, y)\right|+\frac{\omega(f ; \delta)}{\delta^{2}} L_{m, n}(\varphi ; x, y)+\omega(f ; \delta) \\
& +N\left|L_{m, n}\left(f_{0} ; x, y\right)-f_{0}(x, y)\right|,
\end{aligned}
$$

where $N:=\|f\|$. Taking supremum over $(x, y) \in D$ on the both-sides of the above inequality, we obtain, for any $\delta>0$,

$$
\begin{gathered}
\left\|L_{m, n} f-f\right\| \leq \omega(f ; \delta)\left\|L_{m, n} f_{0}-f_{0}\right\|+\frac{\omega(f ; \delta)}{\delta^{2}}\left\|L_{m, n} \varphi\right\| \\
+\omega(f ; \delta)+N\left\|L_{m, n} f_{0}-f_{0}\right\| .
\end{gathered}
$$

Now if we take $\delta:=\delta_{m, n}:=\sqrt{\left\|L_{m, n}(\varphi)\right\|}$, then we may write

$$
\left\|L_{m, n} f-f\right\| \leq \omega(f ; \delta)\left\|L_{m, n} f_{0}-f_{0}\right\|+2 \omega(f ; \delta)+N\left\|L_{m, n} f_{0}-f_{0}\right\|
$$

and hence

(3.3) $\left\|L_{m, n} f-f\right\| \leq D\left\{\omega(f ; \delta)\left\|L_{m, n} f_{0}-f_{0}\right\|+\omega(f ; \delta)+\left\|L_{m, n} f_{0}-f_{0}\right\|\right\}$, where $D=\max \{2, N\}$. For a given $r>0$, define the following sets:

$$
\begin{aligned}
U & :=\left\{(m, n):\left\|L_{m, n}(f)-f\right\| \geq r\right\}, \\
U_{1} & :=\left\{(m, n): \omega(f ; \delta)\left\|L_{m, n} f_{0}-f_{0}\right\| \geq \frac{r}{3 D}\right\}, \\
U_{2} & :=\left\{(m, n): \omega(f ; \delta) \geq \frac{r}{3 D}\right\}, \\
U_{3} & :=\left\{(m, n):\left\|L_{m, n} f_{0}-f_{0}\right\| \geq \frac{r}{3 D}\right\} .
\end{aligned}
$$

It follows from (3.3) that

$$
U \subset U_{1} \cup U_{2} \cup U_{3}
$$

Also define the sets:

$$
U_{4}:=\left\{(m, n): \omega(f ; \delta) \geq \sqrt{\frac{r}{3 D}}\right\},
$$




$$
U_{5}:=\left\{(m, n):\left\|L_{m, n} f_{0}-f_{0}\right\| \geq \sqrt{\frac{r}{3 D}}\right\} .
$$

Then, observe that $U_{1} \subset U_{4} \cup U_{5}$. So, we have $U \subset U_{2} \cup U_{3} \cup U_{4} \cup U_{5}$. Now, since $\gamma_{m, n}:=\max \left\{\alpha_{m, n}, \beta_{m, n}\right\}$ for each $(m, n) \in \mathbb{N}^{2}$, we get for all $(j, k) \in \mathbb{N}^{2}$,

$$
\begin{aligned}
\frac{1}{\gamma_{j, k}} \sum_{(m, n) \in U} a_{j, k, m, n} \leq & \frac{1}{\beta_{j, k}} \sum_{(m, n) \in U_{2}} a_{j, k, m, n}+\frac{1}{\alpha_{j, k}} \sum_{(m, n) \in U_{3}} a_{j, k, m, n} \\
& +\frac{1}{\beta_{j, k}} \sum_{(m, n) \in U_{4}} a_{j, k, m, n}+\frac{1}{\alpha_{j, k}} \sum_{(m, n) \in U_{5}} a_{j, k, m, n} .
\end{aligned}
$$

Letting $j, k \rightarrow \infty$ (in any manner) and using (i) and (ii), we obtain

$$
P-\lim _{j, k} \frac{1}{\gamma_{j, k}} \sum_{(m, n) \in U} a_{j, k, m, n}=0 .
$$

The proof is complete.

The following analog also holds.

Theorem 3. Let $\left\{L_{m, n}\right\}$ be a sequence of positive linear operators from $H_{\omega}(K)$ into $C(K)$, and let $A=\left(a_{j, k, m, n}\right)$ be a nonnegative $R H$-regular summability matrix method. Assume that the following conditions hold:

(i) $\left\|L_{m, n}\left(f_{0}\right)-f_{0}\right\|=s t_{A}^{(2)}-o_{m}\left(\alpha_{m, n}\right)$ as $m, n \rightarrow \infty$,

(ii) $\omega\left(f ; \delta_{m, n}\right)=s t_{A}^{(2)}-o_{m}\left(\beta_{m, n}\right)$ as $m, n \rightarrow \infty$, where $\delta_{m, n}:=\sqrt{\left\|L_{m, n}(\varphi)\right\|}$ with $\varphi(u, v)=\left(\frac{u}{1-u}-\frac{x}{1-x}\right)^{2}+\left(\frac{v}{1-v}-\frac{y}{1-y}\right)^{2}$. Then, for any $f \in H_{\omega}(K)$,

$$
\left\|L_{m, n}(f)-f\right\|=s t_{A}^{(2)}-o_{m}\left(\gamma_{m, n}\right) \text { as } m, n \rightarrow \infty,
$$

where $\gamma_{m, n}:=\max \left\{\alpha_{m, n}, \beta_{m, n}\right\}$ for each $(m, n) \in \mathbb{N}^{2}$. Similar results hold when little "om" is replaced by big " $O_{m}$ ".

\section{References}

[1] G. A. Anastassiou and O. A. Duman, A Baskakov type generalization of statistical Korovkin theory, J. Math. Anal. Appl. 340 (2008), no. 1, 476-486.

[2] _ Statistical fuzzy approximation by fuzzy positive linear operators, Comput. Math. Appl. 55 (2008), no. 3, 573-580.

[3] F. Altomare and M. Campiti, Korovkin-Type Approximation Theory and Its Applications, de Gruyter Studies in Mathematics, 17. Walter de Gruyter \& Co., Berlin, 1994.

[4] R. Bojanic and M. K. Khan, Summability of Hermite-Fejér interpolation for functions of bounded variation, J. Natur. Sci. Math. 32 (1992), no. 1, 5-10.

[5] O. Duman, E. Erkuş, and V. Gupta, Statistical rates on the multivariate approximation theory, Math. Comput. Modelling 44 (2006), no. 9-10, 763-770.

[6] O. Duman, M. K. Khan, and C. Orhan, A-statistical convergence of approximating operators, Math. Inequal. Appl. 6 (2003), no. 4, 689-699.

[7] E. Erkus and O. Duman, A-statistical extension of the Korovkin type approximation theorem, Proc. Indian Acad. Sci. Math. Sci. 115 (2005), no. 4, 499-508. 
[8] E. Erkuş, O. Duman, and H. M. Srivastava, Statistical approximation of certain positive linear operators constructed by means of the Chan-Chyan-Srivastava polynomials, Appl. Math. Comput. 182 (2006), no. 1, 213-222.

[9] H. J. Hamilton, Transformations of multiple sequences, Duke Math. J. 2 (1936), no. 1, 29-60.

[10] G. H. Hardy, Divergent Series, Oxford Univ. Press, London, 1949.

[11] S. Karakuş, K. Demirci, and O. Duman, Equi-statistical convergence of positive linear operators, J. Math. Anal. Appl. 339 (2008), no. 2, 1065-1072.

[12] P. P. Korovkin, Linear Operators and Approximation Theory, Hindustan Publ. Co., Delhi, 1960

[13] F. Móricz, Statistical convergence of multiple sequences, Arch. Math. (Basel) 81 (2003), no. $1,82-89$.

[14] Mursaleen and O. H. H. Edely, Statistical convergence of double sequences, J. Math. Anal. Appl. 288 (2003), no. 1, 223-231.

[15] A. Pringsheim, Zur theorie der zweifach unendlichen zahlenfolgen, Math. Ann. 53 (1900), no. 3, 289-321.

[16] G. M. Robison, Divergent double sequences and series, Trans. Amer. Math. Soc. 28 (1926), no. 1, 50-73.

[17] F. Taşdelen and A. Erençin, The generalization of bivariate $M K Z$ operators by multiple generating functions, J. Math. Anal. Appl. 331 (2007), no. 1, 727-735.

Kamil Demirci

Faculty of Sciences and Arts Department of Mathematics

Sinop University

57000 SiNOP, TURKEY

E-mail address: kamild@sinop.edu.tr

FADIME DIRIK

Faculty of Sciences and Arts Department of Mathematics

SINOP UNIVERSITY

57000 Sinop, Turkey

E-mail address: fgezer@sinop.edu.tr 